------ Raf. J. Sci., Vol. 22, No.4 pp 62-74 , 2011------

\title{
Synthesis and Antibacterial Evaluation of Some New Fused Cyclic Sulfoxides
}

\author{
Tahani W. Jihad \\ Department of Chemistry \\ College of Science \\ University of Mosul \\ E-mail : tahani.waleed@yahoo.com
}

(Received 1/3/2011; Accepted 16/5/2011)

\begin{abstract}
A series of diarylidine cyclohexanones $\left(1_{\mathrm{t}}-12_{\mathrm{t}}\right)$ have been prepared via ClasienSchmidt reaction then condensed with dimethyl sulphoxide through Michael-Claisen condensation to afford the corresponding fused heterocycles $\left(13_{t}-24_{t}\right)$. The structures of the products were elucidated by the spectrum (UV, IR, ${ }^{1} \mathrm{H}-\mathrm{NMR}$ ). The suggested mechanisms for these reactions were investigated according to theoretical calculations, heat of formation (H.F.) and the steric energy (S.E.). Furthermore some of the products have been tested for their biological activity (antibacterial).
\end{abstract}

Keywords: Dimethyl sulfoxide, Diarylidene cyclohexanone, Antibacterial effect, Fused cyclic sulfoxide.

\section{تشييد وقيم الهمالية المضالة للبكتربا لبصض السالفوكسيدات الحافية المنفجة الجسية}

\section{الملغص}

قم تحضيرسلسلة من ثنائي ارايليين هكسانون حلقي (12t - 12 من خلل تفاعل كليزن ثشميدت حيث

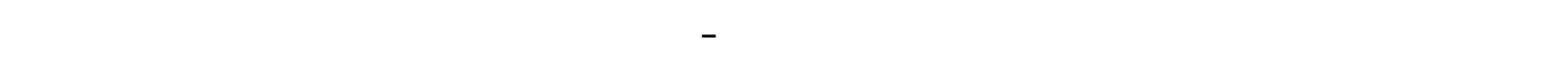

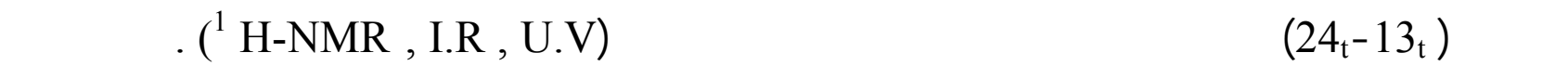
من الميكانيكيت المقترحة لهذه الفاعلات لمتنادا إلى المسابت الظرية لحرارة النكوين (H.F.) وطلة فة فئة

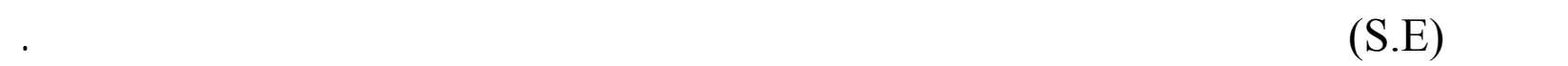




\section{INTODUCTION}

Sulfoxides are compounds that contain a sulfinyl group covalently bonded at the sulfur atom to two carbon atoms and oxygen atom (Segurel et al., 2005). The naturally occurring sulfoxides are often accompained by the corresponding sulfides or sulfones (Lake et al., 1988). The most commercially important sulfoxide is the simplest member, dimethyl sulfoxide. Sulfoxides occur widely in small concentrations in plant and animal tissues (Wiley and Sons, 2001). Sulfoxides have fascinated organic chemists for a long time owning to their varied reactivity as a functional group for transformation into a variety of organo sulfur compounds. These transformations are ureful for the synthesis of drugs and sulfur - substituted natural products (Musah et al., 2009). Optically active sulfoxides continue to deserve much attention as important chiral auxiliaries in asymmetric synthesis and in $\mathrm{C}-\mathrm{C}$ bond forming reactions (Abbas et al., 2005). A novel bioactive sulfoxide like Nacetyl-2-(1-adamantylsulfoxo)-3-acetoxy-4-phenyl-6-hydroxy-1,2,3,6-tetrahydropyridine was tested for anti-microbial action using the agar dilution method against twenty one microorganisms gram - positive and gram - negative bacteria and diploid fungus. It was found that the sulfoxide inhibited the growth of Moraxella catarrhalis and Streptococcus pyogenes with minimum inhibitory concentration.in addition, the growth of Corynebacterium diphtheriae (NCTC) was partially inhibitted (80\%) at $256 \mu \mathrm{g} / \mathrm{ml}$ (Prachayasittikul et al., 2010).

Different routes were used to afford the cyclic sulfoxides such as the condensation of $\mathrm{MeS}(\mathrm{O}) \mathrm{CH}_{2} \mathrm{Na}$ with $\mathrm{phCH}=\mathrm{CHBz}$ in liquid ammonia which gave 3-hydroxy-3,5-diphenyl -1-thiacyclohexane-1-oxide as shown below (Gautier et al., 1970).

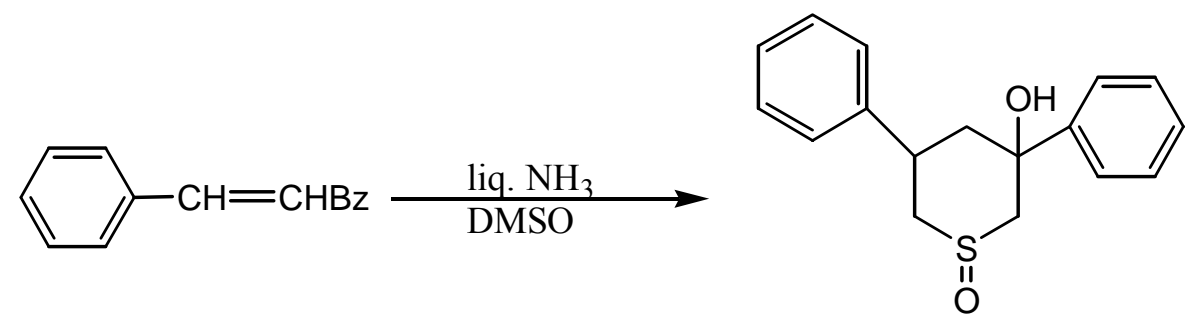

Bz: Benzoyl

Chloroperoxidase was used as catalyst in the synthesis of a series of aromatic cyclic sulfoxides. This have been acheived by the reaction as shown below (Allenmark and Andersson, 1996).

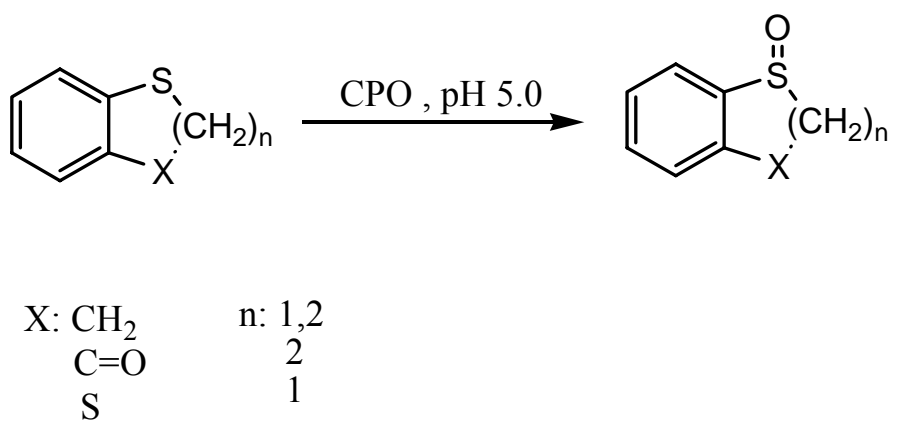


Michael addition of DMSO to permethylated core of oligosaccharides was used in the formation of covalent DMSO products adducts as shown below (Sioud et al., 2010).

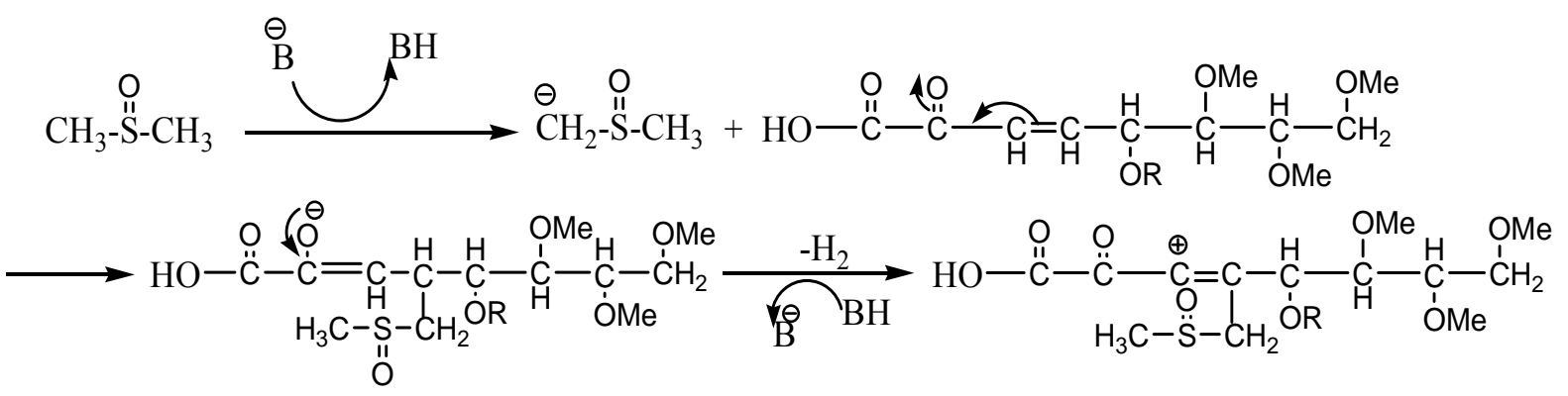

\section{Materials}

\section{EXPERIMENTAL}

All chemicals was supplied from Fluka, Sigma and Aldrich company.

Melting points were determined by Electrothermal 1A 9000 Digital - series 1998 apparatus (uncorrected).

UV-visible spectra were recorded using Shimadzu UV-Vissible spectrophotometer 160 (Department of Chemistry - College of Science - University of Mosul).

Fourier-Transform Infrared spectra were recorded on Brucker Tensor spectrophotometer 2003 (Germany) (Department of Chemistry - College of Science University of Mosul).

Nuclear magnetic resonance $\left({ }^{1} \mathrm{H}-\mathrm{NMR}\right)$ spectra were recorded using, $500 \mathrm{MHz}$ perkin Elmer spectrometer, using tetramethylsilane (TMS) as an internal standard, $\mathrm{CDCl}_{3}$ as a solvent was used in our investigation.( Department of Chemistry- I. I. T. Roorkee, India).

The theoretical calculations based on the data obtained from the minimized geometry were computed using semi-empirical AM1 module in the CS Chem. Office 2003 version 8.0 molecular modeling package.

\section{Starting materials}

I. Diarylidene cyclohexanones $\left(\mathbf{1}_{\mathbf{t}} \mathbf{- 1 2}\right)$ (General procedure ) (Karthikeyan et al., 2009), (Qitto, 2008), (Yonis Mahmood, 2009) and (Al-lewzy, 2011).

These compounds were prepared by stirring $(3.7 \mathrm{mmol})$ of the cyclohexanone with 7.5 mmol of the desired benzaldehyde, and $5 \mathrm{ml}$ of $10 \%$ ethanolic sodium hydroxide in $25 \mathrm{ml}$ ethanol in a $50 \mathrm{ml}$ round-bottomed flask. After 3 hours the reaction mixture was filtered to obtain the crude precipitate which then washed several times with ethanol. The precipitate was dried and recrystallized from appropriate solvent. The colour for all diarylidene cyclohexanones is distinct yellow. (Table-1 and Table-2). 
<smiles>[X]c1ccc(/C=C2\CCC/C(=C\c3ccc([X])cc3)C2=O)cc1</smiles>

Table1: Names and some physical properties of $\left(1_{\mathrm{t}}-12_{\mathrm{t}}\right)$.

\begin{tabular}{|c|c|c|c|c|c|c|}
\hline $\begin{array}{l}\text { Cpd } \\
\text { No. }\end{array}$ & Name & $\mathbf{X}$ & $\begin{array}{l}\text { Recryst. } \\
\text { From }\end{array}$ & $\begin{array}{l}\text { m.p. } \\
\left({ }^{\circ} \mathrm{C}\right)\end{array}$ & $(\text { Lit. })^{*}$ & $\begin{array}{l}\text { Yield } \\
(\%)\end{array}$ \\
\hline $1_{t}$ & $\begin{array}{l}\text { 2,6 - Dibenzylidene } \\
\text { cyclohexanone }\end{array}$ & $\mathrm{H}$ & ethanol & $119-120$ & 116-119 & 24 \\
\hline $22_{t}$ & $\begin{array}{l}\text { 2,6-Di(2-chloro benzylidene) } \\
\text { cyclohexanone }\end{array}$ & $2-\mathrm{Cl}$ & ethanol & $112-113$ & $110-112$ & 22 \\
\hline $3 \mathrm{t}$ & $\begin{array}{l}\text { 2,6-Di(4-methoxy benzylidene) } \\
\text { cyclohexanone }\end{array}$ & $4-\mathrm{OMe}$ & ethanol & $157-158$ & $156-158$ & 18 \\
\hline $4_{t}$ & $\begin{array}{l}\text { 2,6-Di(4-methyl benzylidine) } \\
\text { cyclohexanone }\end{array}$ & $4-\mathrm{Me}$ & ethanol & $163-165$ & ------ & 21 \\
\hline $5 \mathrm{t}$ & $\begin{array}{l}\text { 2,6-Di(2,4-dimethoxy } \\
\text { benzylidene) cyclohexanone }\end{array}$ & 2,4-diOMe & xylene & $170-171$ & 164-166 & 18 \\
\hline $6_{\mathrm{t}}$ & $\begin{array}{l}\text { 2,6-Di(2,4-dichloro benzylidene }) \\
\text { cyclohexanone }\end{array}$ & 2,4-diCl & $\begin{array}{c}\text { carbon } \\
\text { tetra } \\
\text { chloride }\end{array}$ & $161-163$ & ------- & 36 \\
\hline $7 \mathrm{t}$ & $\begin{array}{l}\text { 2,6-Di(3-nitrobenzylidene) cyclo } \\
\text { hexanone }\end{array}$ & $3-\mathrm{NO}_{2}$ & $\begin{array}{c}\text { carbon } \\
\text { tetra } \\
\text { chloride }\end{array}$ & 194 & $190-192$ & 27 \\
\hline $8 \mathrm{t}$ & $\begin{array}{l}\text { 2,6-Di(4-fluoro benzylidene) } \\
\text { cyclohexanone }\end{array}$ & $4-F$ & ethanol & $149-150$ & $154-155$ & 29 \\
\hline $9 \mathrm{t}$ & $\begin{array}{l}\text { 2,6-Di(3-bromo benzylidene) } \\
\text { cyclohexanone }\end{array}$ & $3-\mathrm{Br}$ & ethanol & 113 & $111-113$ & 21 \\
\hline $10_{t}$ & $\begin{array}{l}\text { 2,6-Di(2-piperonylidene) } \\
\text { cyclohexanone }\end{array}$ & $\begin{array}{c}2- \\
\text { piperonyl }\end{array}$ & $\begin{array}{c}\text { carbon } \\
\text { tetra } \\
\text { chloride }\end{array}$ & $181-183$ & ------ & 33 \\
\hline $11_{t}$ & $\begin{array}{l}\text { 2,6-Di(2-furylidene) cyclo } \\
\text { hexanone }\end{array}$ & 2-furyl & ethanol & 142-144 & 143-145 & 35 \\
\hline $12_{t}$ & $\begin{array}{l}\text { 2,6-Di(1-naphthylidene) } \\
\text { cyclohexanone }\end{array}$ & $\begin{array}{c}1- \\
\text { Naphthyl }\end{array}$ & $\begin{array}{c}\text { carbon } \\
\text { tetra } \\
\text { chloride }\end{array}$ & $198-200$ & ------- & 22 \\
\hline
\end{tabular}

*( Yonis Mahmood, 2009) and ( Al-lewzy, 2011) 
Table 2: Spectral data of compounds $\left(1_{t^{-}}-12_{t}\right)$.

\begin{tabular}{|c|c|c|c|c|c|c|c|}
\hline \multirow{2}{*}{$\begin{array}{l}\text { Cpd } \\
\text { No. }\end{array}$} & \multirow[t]{2}{*}{ Structure } & \multirow{2}{*}{$\begin{array}{c}\mathrm{UV}\left(\mathrm{CHCl}_{3}\right) \\
\lambda_{\max }, \mathrm{nm}, \\
\left(\varepsilon \times \mathbf{1 0}^{-3}\right) \\
\text { L.mole } \\
\text {. }^{-1} \mathrm{~cm}^{-1}\end{array}$} & \multicolumn{5}{|c|}{ IR(KBr), $v \mathrm{~cm}^{-1}$} \\
\hline & & & $\mathrm{C}=\mathbf{O}$ & $\mathrm{C}=\mathrm{C}$ & $\begin{array}{c}\mathbf{C}=\mathbf{C} \\
\text { aromatic }\end{array}$ & $\begin{array}{c}\mathrm{NO}_{2} \\
\text { Sym., assym }\end{array}$ & $\begin{array}{c}\text { C-O-C } \\
\text { Sym., assym }\end{array}$ \\
\hline $1_{\mathrm{t}}$ & & $\begin{array}{c}326 \\
(2.752)\end{array}$ & 1661 & 1607 & $\begin{array}{l}1486, \\
1573\end{array}$ & $\ldots \ldots \ldots$ & ......... \\
\hline $2 \mathrm{t}$ & & $\begin{array}{c}336 \\
(2.752)\end{array}$ & 1664 & 1602 & $\begin{array}{l}1469 \\
1575\end{array}$ & f....... & $\ldots \ldots \ldots$ \\
\hline $3 \mathrm{t}$ & & $\begin{array}{c}306 \\
(2.683)\end{array}$ & 1658 & 1595 & $\begin{array}{l}1508 \\
1554\end{array}$ & $\ldots \ldots \ldots$ & 1024,1250 \\
\hline $4_{t}$ & & $\begin{array}{c}326 \\
(2.738)\end{array}$ & 1660 & 1601 & $\begin{array}{l}1508, \\
1565\end{array}$ & $\ldots \ldots \ldots$ & n...... \\
\hline $5 \mathrm{t}$ & & $\begin{array}{c}308 \\
(2.683)\end{array}$ & 1662 & 1597 & $\begin{array}{l}1468, \\
1500\end{array}$ & $\ldots$ & 1027,1207 \\
\hline $6_{t}$ & & $\begin{array}{c}324 \\
(2.767)\end{array}$ & 1660 & 1598 & $\begin{array}{l}1468, \\
1578\end{array}$ & $\ldots \ldots \ldots$ & n..... \\
\hline $7 \mathrm{t}$ & & $\begin{array}{c}340 \\
(2.754)\end{array}$ & 1663 & 1638 & $\begin{array}{l}1565 \\
1615\end{array}$ & 1347,1534 & $\ldots \ldots \ldots$ \\
\hline $8_{\mathrm{t}}$ & & $\begin{array}{c}328 \\
(2.767)\end{array}$ & 1662 & 1608 & $\begin{array}{l}1507, \\
1567\end{array}$ & ........ & $\cdots \cdots \cdots$ \\
\hline $99_{t}$ & & $\begin{array}{c}362 \\
(2.696)\end{array}$ & 1661 & 1606 & $\begin{array}{l}1573, \\
1591\end{array}$ & ........ & ........ \\
\hline $10_{t}$ & & $\begin{array}{c}302 \\
(2.671)\end{array}$ & 1656 & 1588 & $\begin{array}{l}1489 \\
1555\end{array}$ & n..... & 1028,1262 \\
\hline $11_{t}$ & & $\begin{array}{c}328 \\
(2.738)\end{array}$ & 1643 & 1591 & $\begin{array}{l}1547 \\
1560\end{array}$ & $\ldots \ldots \ldots$ & 1020,1248 \\
\hline $12_{\mathrm{t}}$ & & $\begin{array}{c}334 \\
(2.768)\end{array}$ & 1662 & 1604 & $\begin{array}{l}1504 \\
1574\end{array}$ & $\ldots \ldots \ldots$ & \\
\hline
\end{tabular}




\section{The sulfoxide compounds:}

General procedure:( Ghazal, 1997 )

The desired diarylidine cyclohexanone $(1 \mathrm{mmole})$ was allowed to react with $10 \mathrm{ml}$ of dimethylsulphoxide in a $50 \mathrm{ml}$ round-bottomed flask. A drop-wise addition with stirring of $10 \%$ ethanolic $\mathrm{NaOH}$ cause the colour to be changed. Stirring is continued for 5 hours till the colour became dark brown (in case of no colour change the reaction mixture is refluxed for $10 \mathrm{~min}$ ). After cooling, water is added to precipitate the corresponding products which then filtered off, washed with water then dried at r.t. and recrystallized from ethanol. (Table3 and Table-4). It seems that the products were less soluble in DMSO than the reactants hence the reactants remained soluble in the DMSO- $\mathrm{H}_{2} \mathrm{O}$ mixture while the product was precipitated .

Table 3: Names and some physical properties of $\left(13_{\mathrm{t}}-24_{\mathrm{t}}\right)$.

\begin{tabular}{|c|c|c|c|c|c|}
\hline $\begin{array}{l}\text { Cpd } \\
\text { No. }\end{array}$ & Names & $\mathbf{X}$ & $\begin{array}{l}\text { m.p. } \\
\left({ }^{\circ} \mathrm{C}\right)\end{array}$ & $\begin{array}{c}\text { Yield } \\
(\%)\end{array}$ & Colour \\
\hline $13_{t}$ & $\begin{array}{l}\text { 3,4-Dihydro-4-phenyl -8-benzylidene cyclohexano } \\
\text { [1,2-c ]-1-oxo thiapyran }\end{array}$ & $\mathrm{H}$ & $106-110$ & 16 & Brown \\
\hline $14_{t}$ & $\begin{array}{l}\text { 3,4-Dihydro-4-(2-chloro phenyl) -8-(2-chloro } \\
\text { benzylidene ) cyclohexano [1,2-c ]-1-oxo thiapyran }\end{array}$ & $2-\mathrm{Cl}$ & $150-153$ & 12 & $\begin{array}{l}\text { Light } \\
\text { brown }\end{array}$ \\
\hline $15_{t}$ & $\begin{array}{l}\text { 3,4-Dihydro-4-(4-methoxy phenyl) -8-(4-methoxy } \\
\text { benzylidene) cyclohexano [1,2-c ]-1-oxo thiapyran }\end{array}$ & $4-\mathrm{OMe}$ & $116-120$ & 13 & Green \\
\hline $16_{t}$ & $\begin{array}{l}\text { 3,4-Dihydro-4-(4-methyl phenyl) -8-(4-methyl } \\
\text { benzylidene ) cyclohexano [1,2-c ]-1-oxo thiapyran }\end{array}$ & 4-Me & 95-98 & 11 & Brown \\
\hline $17_{\mathrm{t}}$ & $\begin{array}{l}\text { 3,4-Dihydro-4-(2,4-dimethoxy phenyl) -8-(2,4- } \\
\text { dimethoxy benzylidene) cyclohexano }[1,2-c]-1- \\
\text { oxo thiapyran }\end{array}$ & 2,4-diOMe & $80-83$ & 20 & Brown \\
\hline $18_{t}$ & $\begin{array}{l}\text { 3,4-Dihydro-4-(2,4-dichloro phenyl) -8-(2,4- } \\
\text { dichloro benzylidene ) cyclohexano [1,2-c ]-1-oxo } \\
\text { thiapyran }\end{array}$ & 2,4-diCl & $186-186$ & 17 & $\begin{array}{l}\text { Light } \\
\text { brown }\end{array}$ \\
\hline $19_{t}$ & $\begin{array}{l}\text { 3,4-Dihydro-4-(3-nitro phenyl) -8-(3-nitro } \\
\text { benzylidene) cyclohexano [1,2-c ]-1-oxo thiapyran }\end{array}$ & $3-\mathrm{NO}_{2}$ & $158-160$ & 10 & Brown \\
\hline $20_{t}$ & $\begin{array}{l}\text { 3,4-Dihydro-4-(4-fluro phenyl) -8-(4-fluro } \\
\text { benzylidene) cyclohexano [1,2-c ]-1-oxo thiapyran }\end{array}$ & $4-F$ & 104-106 & 10 & Brown \\
\hline $21_{t}$ & $\begin{array}{l}\text { 3,4-Dihydro-4-(3-bromo phenyl) -8-(3-bromo } \\
\text { benzylidene) cyclohexano [1,2-c ]-1-oxo thiapyran }\end{array}$ & $3-\mathrm{Br}$ & $120-123$ & 24 & Brown \\
\hline $22_{t}$ & $\begin{array}{l}\text { 3,4-Dihydro-4-(2-piperonyl) -8-(2-piperonylidene) } \\
\text { cyclohexano [1,2-c ]-1-oxo thiapyran }\end{array}$ & piperonyl & 104-108 & 5 & Orange \\
\hline $23_{t}$ & $\begin{array}{l}\text { 3,4-Dihydro-4-(2-furyl) -8-(2-furylidene) } \\
\text { cyclohexano [1,2-c ]-1-oxo thiapyran }\end{array}$ & 2-Furyl & $182-185$ & 13 & $\begin{array}{l}\text { Light } \\
\text { brown }\end{array}$ \\
\hline $24_{t}$ & $\begin{array}{l}\text { 3,4-Dihydro-4-(1-naphthyl) -8-(1-naphthylidene) } \\
\text { cyclohexano [1,2-c ]-1-oxo thiapyran }\end{array}$ & 1-Naphthyl & $124-126$ & 16 & $\begin{array}{l}\text { Distinct } \\
\text { brown }\end{array}$ \\
\hline
\end{tabular}


Table-4 Spectral data (UV, IR, $\left.{ }^{1} \mathrm{H}-\mathrm{NMR}\right)$ for compounds $\left(13_{t}-24_{t}\right)$

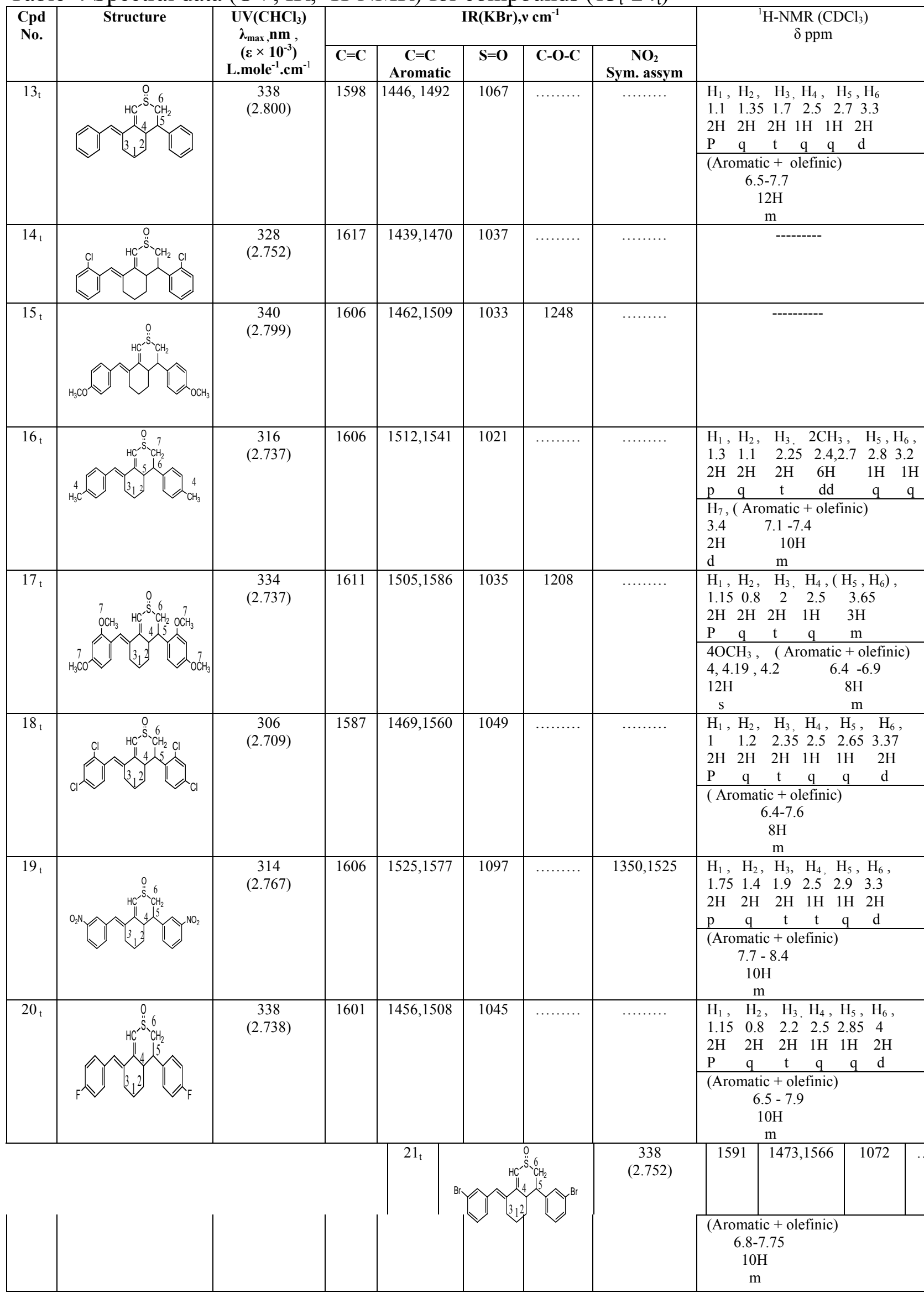




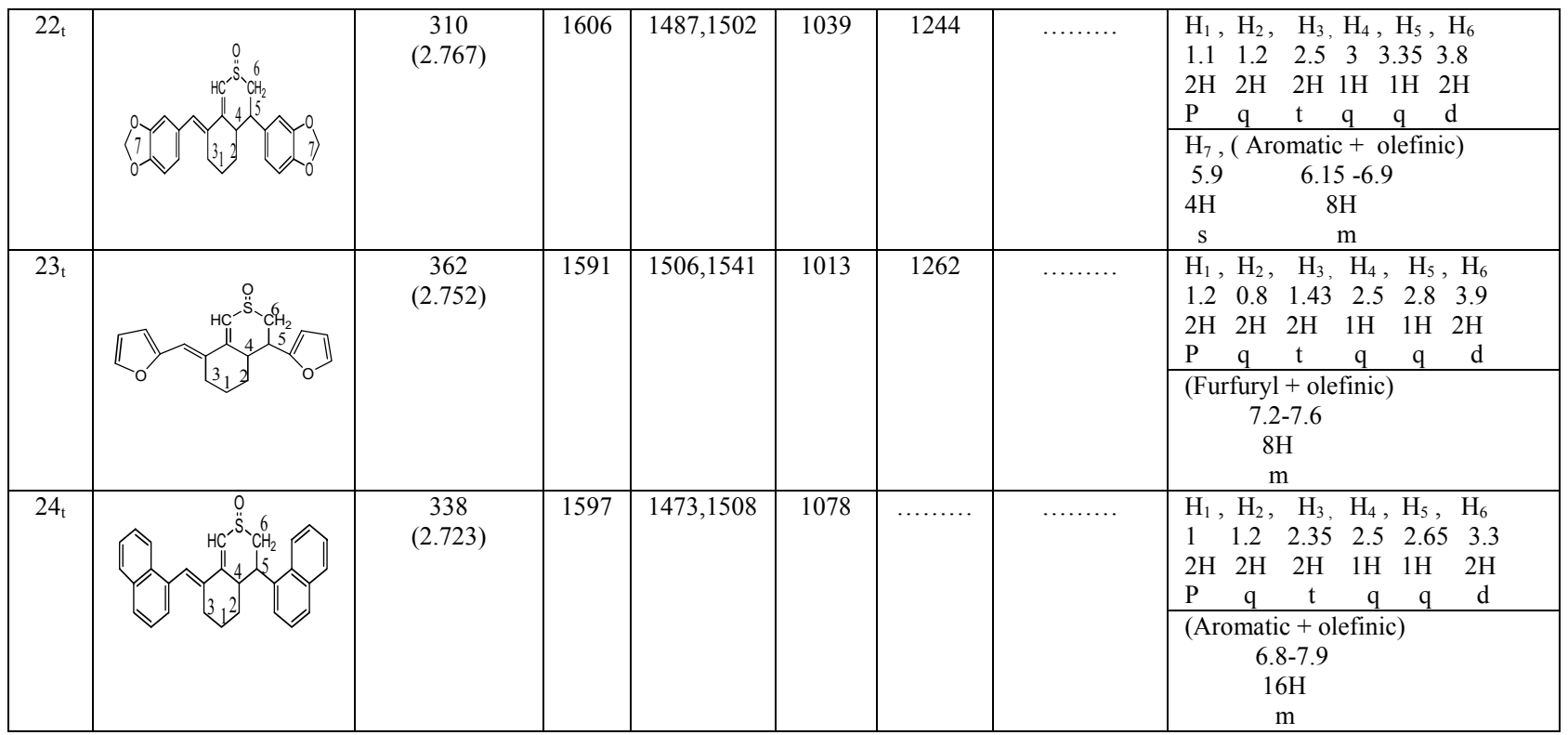

\section{Preliminary biological study:}

In the present work, it is decided to investigate final products and study their inhibitory effect on the growth of two kinds of bacteria Grampositive (staphylococcus aureus) and Gram-negative (E. coli) by using disc diffusion method, (the standard Kirby and Bauer method) (Bauer et al., 1966).

The isolates were isolated and identified in the Dept. of Biology, College of Science, Univ. of Mosul.

\section{PROCEDURE}

Of each bacterial species a loopful were cultured in a nutrient broth and incubated at $37{ }^{\circ} \mathrm{C}$ for $14-16 \mathrm{hr}$, then eventually distributed on the nutrient agar by using a sterile swab. The plates were incubated at $37{ }^{\circ} \mathrm{C}$ for $30 \mathrm{~min}$.

A Whatmann No. 1 type filter paper discs were distributed on the agar and a certain equal $(0.01 \mathrm{mg} / 0.5 \mathrm{ml})$ of the compound per solvent (DMSO) was added. The controls here were Stryptomycin, Vancomycin and Gentamycin for comparison. The plates were then incubated at $37{ }^{\circ} \mathrm{C}$ for 18-24 hr. Prescott ( Prescott et al., 1996 ) method was used to illustrate the sensitivity of the studied compounds. The results were interpreted according to the report of (W.H.O.). The resistance (R) represent the diameter of inhibition zone $<11$ $\mathrm{mm}$, while the sensitive (S) was over $16 \mathrm{~mm}$, but moderately sensitive (MS) was regarded when the inhibition zone is (12-16) $\mathrm{mm}$. 
Table 5: Inhibition effect of compounds $\left(13_{t}-24_{t}\right)$ on growth of Staphylococcus aureus and Escherichia coli.

\begin{tabular}{|c|c|c|}
\hline \multirow{2}{*}{ Compound No. } & \multicolumn{2}{|c|}{ Test organism } \\
\cline { 2 - 3 } & \multirow{2}{|c|}{ E.Coli G(-) } & Sta. Aueus G (+) \\
\hline $13_{\mathrm{t}}$ & $\mathrm{R}$ & $-\mathrm{Ve}$ \\
\hline $14_{\mathrm{t}}$ & $\mathrm{MS}$ & $-\mathrm{Ve}$ \\
\hline $15_{\mathrm{t}}$ & $\mathrm{MS}$ & $-\mathrm{Ve}$ \\
\hline $16_{\mathrm{t}}$ & $\mathrm{R}$ & $-\mathrm{Ve}$ \\
\hline $17_{\mathrm{t}}$ & $-\mathrm{Ve}$ & $-\mathrm{Ve}$ \\
\hline $18_{\mathrm{t}}$ & $\mathrm{R}$ & $-\mathrm{Ve}$ \\
\hline $19_{\mathrm{t}}$ & $-\mathrm{Ve}$ & $-\mathrm{Ve}$ \\
\hline $20_{\mathrm{t}}$ & $\mathrm{R}$ & $-\mathrm{Ve}$ \\
\hline $21_{\mathrm{t}}$ & $-\mathrm{Ve}$ & $-\mathrm{Ve}$ \\
\hline $22_{\mathrm{t}}$ & $-\mathrm{Ve}$ & $-\mathrm{Ve}$ \\
\hline $23_{\mathrm{t}}$ & $-\mathrm{Ve}$ & $-\mathrm{Ve}$ \\
\hline $24_{\mathrm{t}}$ & $\mathrm{MS}$ & $-\mathrm{Ve}$ \\
\hline Control & & $\mathrm{S}$ \\
\hline gentamycin & $\mathrm{S}$ & $\cdots \cdots$ \\
\hline CDz30 & $-\mathrm{Ve}$ & $\mathrm{S}$ \\
\hline (cefodizime) & $\ldots \ldots \ldots$ & \\
\hline Tetracycline & & \\
\hline Retr|
\end{tabular}

R: Resistant

MS: Moderately sensitive

S: Sensitive

\section{RESULTS AND DISCUSSION}

The structures of the starting materials $\left(1_{\mathrm{t}}-12_{\mathrm{t}}\right)$ and the products $\left(13_{\mathrm{t}}-24_{\mathrm{t}}\right)$ were confirmed by (UV, IR) more over the structure of the final products were confirmed by ( $\left.{ }^{1} \mathrm{H}-\mathrm{NMR}\right)$ (see Tables 1-4). The biological activities of the final products $\left(13-24_{t}\right)$ had been investigated for two type of bacteria and compared with some common antibiotics like (Gentamycin, Cefodizime and Tetracycline). Compounds number $\left(13_{t}, 14_{t}, 15_{t}, 16_{t}, 18_{t}, 20_{t}\right.$ and $24_{t}$ ) showed inhibitory effects towards E. coli only, but they showed no effects towards Sta. Aueus. Also compounds $\left(17_{t}, 19_{t}, 21_{t}, 22_{t}\right.$, and $\left.23_{t}\right)$ did n't show any inhibitory effects towards Sta. Aueus and E. coli (Table -5).

According to the spectral data obtained on Tables 1-4, the suggested mechanism for the reaction of diarylidine cyclohexanones with dimethylsulphoxide (Scheme-1) may proceed via Michael or Claisen routes as indicated in the (scheme-1) (Qitto, 2008).

\section{i- Michael route}

The anion $\mathrm{H}_{2}{ }^{\ominus} \mathrm{CSOCH}_{3}$ may attack the $\beta$-carbon of the cyclohexanone . 
Table 6: The heat of formation (H.F.) and steric energy (S.E.) for compounds $\left(13_{t}-24_{t}\right)$.

\begin{tabular}{|c|c|c|c|c|}
\hline $\begin{array}{l}\text { Cpd } \\
\text { No. }\end{array}$ & $\mathbf{X}$ & Form & $\begin{array}{l}\text { Heat of formation H.F. } \\
\text { (Kcal/mole) }\end{array}$ & $\begin{array}{c}\text { Steric energy S.E. } \\
\text { (Kcal/mole) }\end{array}$ \\
\hline \multirow[t]{2}{*}{$13_{\mathrm{t}}$} & \multirow[t]{2}{*}{$\mathrm{H}$} & $\mathrm{a}$ & 32.563559 & 10.1849 \\
\hline & & $\mathrm{b}$ & 36.843252 & 11.5606 \\
\hline \multirow{2}{*}{$14_{t}$} & \multirow{2}{*}{$2-\mathrm{Cl}$} & $\mathrm{a}$ & 21.395082 & 11.2858 \\
\hline & & $\mathrm{b}$ & 24.905872 & 11.9686 \\
\hline \multirow[t]{2}{*}{$15_{t}$} & \multirow[t]{2}{*}{ 4-OMe } & $\mathrm{a}$ & -43.286278 & 21.9422 \\
\hline & & $\mathrm{b}$ & -39.008496 & 23.8322 \\
\hline \multirow{2}{*}{$16_{t}$} & \multirow[t]{2}{*}{ 4-Me } & $\mathrm{a}$ & 18.446187 & 10.1619 \\
\hline & & $\mathrm{b}$ & 21.341061 & 11.4784 \\
\hline \multirow[t]{2}{*}{$17_{t}$} & \multirow[t]{2}{*}{ 2,4-DiOMe } & $\mathrm{a}$ & -64.28304 & 36.1100 \\
\hline & & $\mathrm{b}$ & -113.373465 & 37.2457 \\
\hline \multirow[t]{2}{*}{$18_{t}$} & \multirow[t]{2}{*}{ 2,4-DiCl } & $\mathrm{a}$ & 8.262933 & 15.9798 \\
\hline & & $\mathrm{b}$ & 11.422312 & 16.0670 \\
\hline \multirow[t]{2}{*}{$19_{t}$} & \multirow[t]{2}{*}{$3-\mathrm{NO}_{2}$} & $\mathrm{a}$ & 41.012672 & 2.2045 \\
\hline & & $\mathrm{b}$ & 43.672064 & 12.5437 \\
\hline \multirow[t]{2}{*}{$20_{t}$} & \multirow[t]{2}{*}{$4-F$} & $\mathrm{a}$ & -57.867521 & 10.2825 \\
\hline & & $\mathrm{b}$ & -53.948069 & 11.0288 \\
\hline \multirow[t]{2}{*}{$21_{t}$} & \multirow[t]{2}{*}{$3-\mathrm{Br}$} & $\mathrm{a}$ & 42.539050 & 11.3248 \\
\hline & & $\mathrm{b}$ & 46.336908 & 12.2099 \\
\hline \multirow[t]{2}{*}{$22_{t}$} & \multirow[t]{2}{*}{ 2-Piperonyl } & $\mathrm{a}$ & -84.607943 & 29.0593 \\
\hline & & $\mathrm{b}$ & -80.517700 & 29.0497 \\
\hline \multirow[t]{2}{*}{$23_{t}$} & \multirow[t]{2}{*}{ 2-Furyl } & $\mathrm{a}$ & -3.943829 & 28.8390 \\
\hline & & $\mathrm{b}$ & -0.790590 & 28.7838 \\
\hline \multirow[t]{2}{*}{${ }_{\mathrm{t}}^{\theta 24}$} & \multirow[t]{2}{*}{ 1-Naphthyl } & $\mathrm{a}$ & 88.080591 & 2.4750 \\
\hline & & $\mathrm{b}$ & 77.862936 & 6.2183 \\
\hline
\end{tabular}

From the values of the heats of formation and steric energy (Table-6) it was found that (a) is more stable than (b) (scheme 1), But experimentally according to the spectral data of ${ }^{1} \mathrm{H}-\mathrm{NMR}$ it was found that (b) is more favourable than (a), and hence will predominate. It seems that compounds (a) were the thermodynamically (more stable) product while compound (b) are the kinetically (more stable) product. i.e. the activation energy of path (b) is less than path (a), and hence kinetic products were predominate.

\section{ii-Claisen route}

The anion ${ }^{\ominus} \mathrm{CH}_{2} \mathrm{SOCH}_{3}$ may attack the carbonyl carbon via Claisen condensation to afford the intermediate $\mathrm{C}_{1} \cdot\left(\mathrm{C}_{1}\right)$ may cyclize via intramolecular Michael condensation to give $\mathrm{M}_{2} \mathrm{C}_{2}$ or lose a water molecule to afford $\mathrm{C}_{2}$ which in turn may cyclize via intramolecular Michael condensation to produce $b$. Theoretically it may be concluded that the better suitable route is $\mathrm{M}_{1} \rightarrow \mathrm{M}_{2} \mathrm{C}_{2} \rightarrow \mathrm{a}$, rather than $\mathrm{C}_{1} \rightarrow \mathrm{C}_{2} \rightarrow \mathrm{b}$ (Table 5).

\section{Spectroscopic analysis :}

The compound $\left(13_{t}\right)$ is chosen as a representative model for the spectral $\left({ }^{1} \mathrm{H}-\mathrm{NMR}\right.$ and IR) discussion.

The ${ }^{1} \mathrm{H}-\mathrm{NMR}$ spectrum of compound $\left(13_{\mathrm{t}}\right)$ showed a pentet signal at $\delta 1.1 \mathrm{ppm}$ related to the $2 \mathrm{H}$ of $\mathrm{H}_{1}$ whereas the quartet signal at $\delta 1.35 \mathrm{ppm}$ is due to the $2 \mathrm{H}$ at $\mathrm{H}_{2}$. The $2 \mathrm{H}$ at 
$\mathrm{H}_{3}$ seemed a triplet signal at $\delta 1.7 \mathrm{ppm}$. But the quartet signal at $\delta 2.5 \mathrm{ppm}$ is due to the $1 \mathrm{H}$ at $\mathrm{H}_{4}$. Another quartet signal at $\delta 2.7 \mathrm{ppm}$ it seemed to the $1 \mathrm{H}$ at $\mathrm{H}_{5}$. The doublet signal at $\delta 3.3 \mathrm{ppm}$ correspond to $2 \mathrm{H}$ at $\mathrm{H}_{6}$. Finally a multiplet signal at $\delta 6.5-7.7 \mathrm{ppm}$ related to the $12 \mathrm{H}$ of aromatic and olefinic protons. (Table 4 )

The IR spectrum of compound $\left(13_{\mathrm{t}}\right)$ showed a band at $1598 \mathrm{~cm}^{-1}$ related to the stretching vibration of olefinic $(C=C)$. A frequency at $1492 \mathrm{~cm}^{-1}, 1466 \mathrm{~cm}^{-1}$ related to the symmetrical and a symmetrical vibrations of the aromatic $(\mathrm{C}=\mathrm{C})$. But the frequency at 1067 $\mathrm{cm}^{-1}$ related to the sulfoxide group.

The UV spectrum of the products reflects a wavelength at maximum absorption $\left(\lambda_{\max }\right)$ at (306-362) $\mathrm{nm}$ and values the molar absorbtivity at range $\left(2.709-2.88 \times 10^{3}\right)$ L.mole ${ }^{-1} \cdot \mathrm{cm}^{-1}$ that related to the electronic transition $\left(n \rightarrow \pi^{*}\right)$ and $\left(\pi \rightarrow \pi^{*}\right)$.

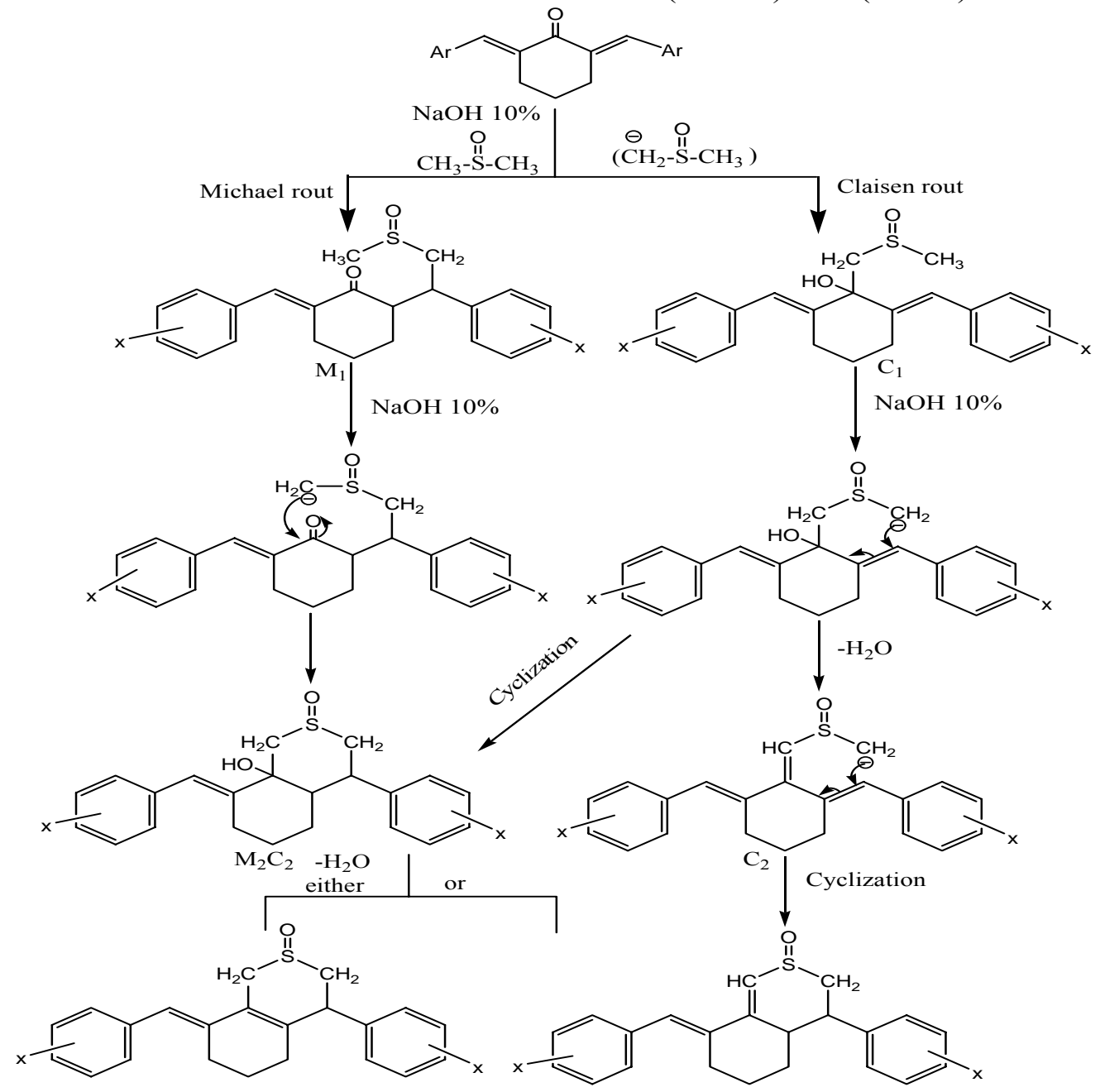

(a)

(b)

$$
\begin{gathered}
\mathrm{Ar}=\mathrm{ph}, \text { 2-furyl, biphenyl, 2- piperonyl, m- } \mathrm{NO}_{2} \mathrm{C}_{6} \mathrm{H}_{4}, 2-\mathrm{Cl} \mathrm{C}_{6} \mathrm{H}_{4}, 2,4-\mathrm{diCl} \mathrm{C}_{6} \mathrm{H}_{3}, 2,4- \\
\text { diOMe } \mathrm{C}_{6} \mathrm{H}_{3}, \mathrm{p}-\mathrm{OMe} \mathrm{C}_{6} \mathrm{H}_{4}, \mathrm{p}-\mathrm{CH}_{3} \mathrm{C}_{6} \mathrm{H}_{4}, \mathrm{p}-\mathrm{F} \mathrm{C}_{6} \mathrm{H}_{4}, \mathrm{~m}-\mathrm{Br} \mathrm{C}_{6} \mathrm{H}_{4}
\end{gathered}
$$

Scheme 1: The mechanistic route of the condensation of diarylidene cyclohexanone with dimethylsulfoxide. 


\section{Acknowledgement}

The author appreciate the recording of Nuclear magnetic resonance spectrum which were recorded in (I.I.T. Roorkee - Department of Chemistry) by Dr. Fawzi Habeeb Jabrail, Department of Chemistry, Collage of Science, University of Mosul. Also the measurements of biological activities by Mrs. Shababa Abdul-Lateef, Department of Biology, College of Science, University of Mosul are gratefully acknowledged.

\section{REFERENCES}

Abbas, S.; Esmael, R.; Masoomeh, Z. (2005). A Solvent free route to the synthesis of diaryl sulfoxides . J. Chem. Chem. Eng., 24( 3), 47.

Allenmark, S.G.; Andersson, M.A. (1996). Chloroperoxidase-Catalyzed asymmetric synthesis of series of aromatic cyclic sulfoxides. Tetrahedron: Asymmetry., 7, 10891094.

Al-lewzy, M.S. (2011). Synthesis and antibacterial evaluation of some fused five and six membered heterocycles, University of Mosul, M.Sc. Thesis, 40.

Bauer, A.W.; Kirby, W. M. M.; Sherris, J.C.; Turk, M.(1966). Antibiotic susceptibility testing by a standarized single disc method. A. J. Clin. Pathol.45, 493-496.

Gautier, A.J.; Marcel, M.; Michel, P.; Henri, M.; Janine, B. (1970). Cyclic sulfoxide obtained by the condensation of benzal acetophenone with dimethyl sulfoxide. Chem. Abst., 72, 7-9, 305, 31548a .

Ghazal, A.Y. (1997). phase transfer catalysis applications for some addition reactions on chalcones, University of Mosul, M.Sc. Thesis. 33-34.

Karthikeyan, N. S.; Sathiyanarayanan, K. I.; Aravindan, P.G. (2009). Ammonium Acetate: An Efficient reagent for the one-pot synthesis of 5-Aryl-7,8,13,14tetrahydrodibenzo [a,i] phenanthridines, 2,4-Diaryl-6,7-bezo-3-azabicyclo[3.3.1] nonan-9-ones and $\alpha, \alpha$ Bis (substituted benzylidene ) cycloalkanones. Bull. Korean Chem. Soc., 30 (11), 2555-2558.

Lake, R. J.; Brennan, M. M.; Blunt, J.W.; Munro, M. H. G.; Pannell, L. K. (1988). Eudistomin $\mathrm{K}$ sulfoxide-an antiviral sulfoxide from the new zeal and ascidian ritterella sigillinoides. Tetrahedron Letters., 29, 2255-2256 .

Musah, R ; Quan He; Kubec, R.; Jadhav, A. (2009). Studies of movel cysteine sulfoxide lyase from petiveria alliacea: the first heteromeric alliinase. American Society of Plant Biologists., 151, 1340-1316.

Prachayasittikul, S.; Pingaew, R.; Worachartcheewan, A.; Ruchirawat, S.; Prachayasittikul, V. (2010). Original article: A New sulfoxide analog of 1,2,3,6Tetrahydrophenylpyridine and antimicrobial activity. Excll J., 9, 102-107.

Prescott, L.M.; Harley, J.P.; Klein, D.A. (1996). "Microbiology ", 3rd edn., Inc. Wm. C. Brown publisher, London Chicago, 436-450.

Quito, T. W. J. ( 2008). " Synthesis of Some Fused Heterocycles and Study Their Expected Biological Activity, University of Mosul, M.Sc .Thesis. p.105.

Segurel, M.A.; Razungles, A. J.; Riou, C.; Trigueiro, M.G.L.; Baumes, R.L. (2005). Ability of possible DMS precursors to release DMS during wine aging and in the conditions of heat-alkaline treatment. J. Agricultural and Food Chem., 53 (7), 2637-2645. 
Sioud, S.; Jahouh, F.; Nashed, M.; Joly, N.; Banoub, J.H. (2010). Determination of distinctive carbohydrate signatures obtained from the aeromonas hydrophila (chemotype II) core oligosaccharide pinpointing the presence of the 4-Ophosphorylated 5-O-linked Kdo reducing end group using electrospray ionization quadrupole orthogonal time-of-flight mass spectrometry and tandem mass spectrometry. Rapid Communication in Mass Spectrometry., 24, 2475-2490.

Wiley, J.; Sons , Inc. (2001). Kirk- Othmer Encyclopedia of Chemical Technology.

Yonis, M. D. S. (2009). Synthesis of bis spiropyrrolidinyl cyclic ketones and biological evaluation of some of them, University of Mosul, M.Sc. Thesis, 31. 